\title{
Inhalt
}

\author{
Vorwort des Herausgebers
}

\section{Teil I:}

Briefe, Tagebuch, Dokumente

a) Der Erste Weltkrieg und die Anfänge der Republik. Letzte Schuljahre. Januar 1918 - März $1923 \ldots . . \ldots$. . . . . . . . . 9

1. An Julie Bonhoeffer, Berlin, 23. 1. 1918 . . . . . . . 9

2. An Ursula Bonhoeffer, Boltenhagen, 17. 6. $1918 \quad 10$

3. An Julie Bonhoeffer, Boltenhagen, 3. 7.1918 . . . . . . 12

4. An Hans Christoph von Hase, Berlin, 15. 7.1918 . . . 13

5. An die Geschwister und Maria Horn, Waldau, 12. 9. $1918 \quad 14$

6. An die Eltern, Waldau, 30. 9. 1918 . . . . . . . . . 16

7. An Julie Bonhoeffer, Berlin, 8. 12.1918 . . . . . . . 16

8. A n Julie Bonhoeffer, Berlin, 25. 12. 1918 . 18

9. An Julie Bonhoeffer, Berlin, 11. 1. 1919 . . . . . . . 19

10. An Julie Bonhoeffer, Berlin, 10. 2. 1919 . . . . 20

11. An die Eltern, Berlin, 20. 5. 1919 . . . . 21

12. An Julie Bonhoeffer, Berlin, 15. 8. 1919 . . . . . . 22

13. An Julie Bonhoeffer, Berlin, 22. 12. 1919 . . . . . . . 23

14. An Julie Bonhoeffer, Berlin, Weihnachten 1919 . 24

15. An Klaus Bonhoeffer, Friedrichsbrunn, 20. 7. 1920 . . . . 25

16. An die Eltern, Friedrichsbrunn, 29. 7. $1920 \quad 27$

17. An die Eltern, Berlin, 23. 8. 1920 . . . . . . . . . . 29

18. An die Eltern, Berlin, 1. 9.1920 . . . . . . . . . . 30

19. Konfirmationsschein, Berlin, 1. 3.1921 .. 31

20. Von Richard Czeppan, Ratibor, 15. 3. 1921 . . . . . . . . 31

21. A n Julie Bonhoeffer, Berlin, 7. 4. 1921 . . . 33

22. Von Klaus Bonhoeffer, Heidelberg, SS 1921 . . . . . . . 34

23. An Julie Bonhoeffer, Berlin, 3. 5. 1921 . . . . . . . . 35

24. An die Eltern, Berlin, Ende August 1921 . 37

25. An Sabine Bonhoeffer, Berlin, 23. 5. 1922 . . . . . . . 39

26. An die Eltern, Berlin, 7. 6. 1922 . . . . . 41

27. An Sabine Bonhoeffer, Berlin, 25. 6. 1922 . . . . . . 43

28. An die Eltern, Tübingen, 7. 7.1922 . . . . . . . . 44

29. An Hans von Dohnanyi, Tübingen, 19. 7. 1922 . 45 
30. An Julie Bonhoeffer, Berlin, 18. 8. 1922 . . . . . . . . . . 46

31. Abiturzeugnis, Berlin, 1. 3. 1923 . . . . . . . . . 47

b) Studentenleben in Tübingen. April 1923 - Februar 1924 . .

32. An die Eltern, Tübingen, Ende April 1923 . . . . . 49

33. An die Eltern, Tübingen, Anfang Mai 1923 . $\quad 50$

34. An die Eltern, Tübingen, 2. Hälfte Mai 1923 . . . . . . 52

35. An die Eltern, Tübingen, Ende Mai 1923

36. Lebenslauf, Tübingen, SS $1923 \ldots \ldots$. . . . . . . . . . . 55

37. An die Eltern, Tübingen, 7. 6. 1923 . . . . . . . . 55

38. An Paula Bonhoeffer, Tübingen, 19. 7. 1923

39. An die Eltern, Friedrichsbrunn, 16. 8.1923 . . . . . . 57

40. An die Eltern, Friedrichsbrunn, 23. 8. 1923 . . 59

41. An die Eltern, Tübingen, 27. 10.1923 . . . . . . . 60

42. An die Eltern, Tübingen, 3. 11. $1923 \ldots$. . . . . . . 61

43. An Helene Yorck von Wartenburg, Tübingen, 3. 11. 1923.

44. An die Eltern, Tübingen, 8. 11.1923 . . . . . . . . . 65

45. An Karl Bonhoeffer, Tübingen, 14. 11. 1923 . . . 66

46. An die Eltern, Ulm, 16. 11. 1923 . . . 68

47. An die Eltern, Ulm, 20. 11. 1923 . . . . . . . . . . 69

48. An Sabine Bonhoeffer, Ulm, 24. 11. 1923 . . . . . 70

49. An die Eltern, Ulm, 25. 11. 1923 . . . . . . . 71

50. An die Eltern, Tübingen, 1. 12.1923 . . . . . . . . . 72

51. An Paula Bonhoeffer, Tübingen, 5. 12. 1923

52. An Sabine Bonhoeffer, Tübingen, ca. 10. 12. 1923 . . . . 75

53. An die Eltern, Tübingen, 12. 12. 1923 . . . . . . . 76

54. An Sabine Bonhoeffer, Tübingen, 5. 2. 1924

55. An die Eltern, Tübingen, 12. 2. 1924 . . . . . . . . . 78

56. An die Eltern, Tübingen, 18. 2. 1924 . . . . 79

c) Italienische Reise. April-Juni 1924 .

57. Italienisches Tagebuch, Rom, Sizilien, Tripoli, 3.4.-4.6.1924 . . . . . . . . . . . . . . 81

58. An die Eltern, Rom, 6. 4. 1924 . . . . . 112

59. An die Eltern, Rom, 16. 4. 1924 . . . . . . . . . 113

60. An Sabine Bonhoeffer, Rom, 16. 4. 1924 . . . . . . 113

61. An die Eltern, Rom, 19. 4. 1924 . . 114

62. An die Eltern, Palermo, 22. 4. 1924 . . . . . . . 116 
63. An Sabine Bonhoeffer, Agrigent, 28. 4. 1924 . 117

64. Von Klaus Bonhoeffer an die Eltern, Tripoli, 5. 5. $1924 \quad$. 118

65. An die Eltern, Tripoli, 5. 5. 1924 . . . . 120

66. An die Eltern, Tripoli, 9. 5. 1924 . 122

67. An die Eltern, Tripoli, 10. 5. 1924 . . . . . . . 126

68. An die Eltern, Rom, 17. 5. 1924 . . . . . . . . 126

69. An Sabine Bonhoeffer, Rom, 17. 5. $1924 \quad$. . . 127

70. An Julie Bonhoeffer, Rom, Mitte Mai 1924 . $\quad$. . . 128

71. An die Eltern, Rom, 21. 5. 1924 . . . . . 129

72. An Hans von Dohnanyi, Rom, Ende Mai 1924 . . . . 129

73. An Sabine Bonhoeffer, Rom, 22. 5. 1924 . 130

74. Von Theodor Pfizer, Tübingen, Mai 1924 . . . . . 131

75. Von Richard Czeppan, Berlin, 23. 5. 1924 . . . . 132

76. An die Eltern, Rom, 27. 5. 1924 . . . . 134

77. An die Eltern, Rom, 31. 5. 1924 . . . . . . . . . . 135

78. An Klaus Bonhoeffer, Siena, 4. 6. 1924 . $\quad$. . 136

79. An die Eltern, Mailand, 6. 6. 1924 . . . . . 136

d) Berliner Studienjahre.

Juni 1924 - Juli 1927 . . . . . . . . . . . . . . . 137

80. An Sabine Bonhoeffer, Berlin, Juni 1924 . . . . . 137

81. Von Wilhelm Dreier, Tübingen, 24. 7. 1924 . $\quad$. 139

82. An Paula Bonhoeffer, Berlin, 5. 8. 1924 . . . . . . . . 141

83. Von Wilhelm Dreier, Freudenstadt, 8. 8. 1924

84. An die Eltern, Berlin, 20. 8. 1924 . . . . . . . . . . 144

85. Von Theodor Pfizer, Gülzow, 21. 8. 1924 . . . . . . . 146

86. An die Eltern, Flensburg, 30. 9. 1924 . . . . . 147

87. An Richard Czeppan, Nordfriesland, Anfang Oktober 1924 . 148

88. Andie Eltern, Sylt, 10. 10. 1924 . . . . 148

89. Von Robert Held, Stuttgart, 23. 3. 1925 . . . 150

90. An die Eltern, Lesum, 25. 8. 1925 . . . . . . . . . 154

91. Von Karl Bonhoeffer, Berlin, 30. 8. 1925

92. Von Paula Bonhoeffer, Berlin, 31. 8. 1925 . . . . . 155

93. An die Eltern, Berlin, 21. 9. 1925 . . . . . . . . 156

94. Von Richard Widmann, Nufringen, 17. 11. 1925 . . . 157

95. Von Richard Widmann, Nufringen, 25. 2. 1926 . . . . . . . 159

96. Von Richard Widmann, Nufringen, 13. 3. 1926 . 161

97. Von Richard Widmann, Nufringen, 29. 4. 1926 . . . . 163

98. An Susanne Bonhoeffer, Gardasee, 19. 8. 1926 . $\quad 167$

99. Von Paula Bonhoeffer, Kampen/Sylt, 26. 8. 1926 . 167

100. An die Eltern, Berlin, 22. 3. 1927 . . . . . . . . . . . 168

101. Von Susanne Bonhoeffer, Heidelberg, 24. 3. 1927 . . . . 170

102. An Karl Bonhoeffer, Friedrichsbrunn, 26. 3. 1927 
e) Promotion und Erstes Theologisches Examen.

Juli 1927 - Januar 1928 .

103. An die Theologische Fakultät, Berlin, Juli 1927 . . . . . . .

104. Lebenslauf, Berlin, Juli 1927 . . . . . . . . . . . .

105. Reinhold Seebergs Gutachten zur Dissertation, Berlin, Juli 1927

106. Gesuch um Zulassung zum Ersten Theologischen Examen, Berlin, 13.9.1927 . . . . . . . . . . . . . 177

107. Liste der Anlagen zum Gesuch, Berlin, 13. 9. 1927 . . . . . . 178

108. Lebenslauf, Berlin, 13. 9. 1927 . . . . . . . . . . . 178

109. Bescheinigung über Teilnahme am Gemeindeleben, Berlin, Herbst 1927. . . . . . . . . . . . . . . . . 179

110. An das Konsistorium, Berlin, 8. 11. 1927 . . . . . . . . . 179

111. Karl Bonhoeffer an Reinhold Seeberg, Berlin, 5. 12. 1927 . . 180

112. An das Konsistorium, Berlin, 7. 12. 1927 . . . . . . . . 181

113. Promotionsurkunde, Berlin, 17. 12. 1927 . . . . . . . . 181

114. Zeugnis des Ersten Theologischen Examens, Berlin, 17. 1. $1928 \quad 184$

115. Bescheinigung über Aufnahme in die Kandidatenliste, Berlin, 17. 1. 1928 . . . . . . . . . . . . . . . 188

116. Vom Konsistorium, Berlin, 28. 1. 1928 . . . . . . . . . . 189

\section{Teil II:}

Arbeiten, Referate, Notizen .............

\section{a) Arbeiten aus der Schulzeit. Februar 1920 - Januar 1923}

1. Referat über Deutschlands Stellung vor dem Ersten Weltkrieg .

2. Hausarbeit zum Abitur über Catull und Horaz . . . . . . . .

b) Arbeiten zur theologischen Orientierung. 1925 . . 219

3. Zwei Notizen zu Schleiermachers Reden über die Religion . 219

4. Seminararbeit über den I. Clemensbrief . . . . . . . . . 220

5. Seminararbeit über Luthers Stimmungen gegenüber seinem Werk ................... . 2 271

6. Referat über historische und pneumatische Schriftauslegung . 305

7. Notiz zu Luthers Römerbriefvorlesung . 324

8. Referat über Vernunft und Offenbarung . . . . . . . . . 325 
c) Arbeiten zum 3. Artikel im Umfeld der Dissertation. Januar - November $1926 \ldots \ldots 336$

9. Referat über Kirche und Eschatologie . . . . . . . . . . . . . 336

10. Seminararbeit über den Heiligen Geist bei Luther . . . . . . . 355

11. ,Freude' im Urchristentum. Festgabe für Adolf von Harnack . 410

12. Referat über die altlutherische Eschatologie . . . . . . . . . . 430

13. Referat über Johannes und Paulus . . . . . . . . . . . . . . . 441

d) Arbeiten in der Schlußphase des Studiums, Promotion und Erstes Theologisches Examen. Januar 1927 - Januar 1928 . . . . . . . . . . . . . 452

14. Seminararbeit über Hiob . . . . . . . . . . . . . . . 452

15. Notizen zu Karl Barths Christlicher Dogmatik . . . . . . . . 473

16. Promotionsthesen . . . . . . . . . . . . . 476

17. Dogmatische Examensklausur über Heilsgewißheit . . . . . . 479

\section{Teil III:}

Predigten, Katechesen, Ansprachen

a) Gemeindepredigten und Ansprachen im Kindergottesdienst vor der Teilnahme an praktisch-theologischen Seminaren.

Oktober 1925 - Mai 1926 . . . . . . . . . . . . . . 485

1. Predigt über Lukas $17,7-10 \ldots \ldots$. . . . . . . . . . . . 485

2. Ansprache über den Dekalog . . . . . . . . . . . . . . . 491

3. Ansprache über das erste Gebot . . . . . . . . . . . . . . 498

4. Ansprache über Matthäus 21,28-31

Die ungleichen Söhne . . . . . . . . . . . . . . . . 502

5. Predigt über Psalm 127,1 . . . . . . . . . . . . . . . 510

b) Texte aus dem homiletischen und dem katechetischen Seminar. SS $1926 \ldots \ldots$. . . . . . . . . . . . . . . 517

6. Exegese und Katechese über Lukas 9,57-62 _ . . . . . . . 517

7. Exegese und Predigt über Jakobus $1,21-25$
Seid Täter des Worts! . . . . . . . . . . . . . . . . 533

8. Katechetischer Entwurf über den zweiten Glaubensartikel . . . 546 
c) Ansprachen im Kindergottesdienst.

November 1926-April 1927 .

9. Ansprache über Lukas $12,35 \mathrm{ff}$

Zum Totensonntag . . . . . . . . . . . . .

10. Ansprache über Psalm 24,7

Zum Advent . . . . . . . . . . . . . . . . . . . . . . . 559

11. Ansprache über Jeremia 27-28 . . . . . . . . . . . . . . . . . 566

12. Ansprache über Johannes 19 . . . . . . . . . . . . . . 573

d) Referat und Katechese für Jugendliche, Examenspredigt. April-Oktober 1927 . . . . . . . . . . . . . . . . 578

13. Einladung zum ,Donnerstagskreis‘ . . . . . . . . . . . . 578

14. Referat über,Die katholische Kirche` für den,Donnerstagskreis‘ 579

15. Meditation und Katechese über die ,Ehre' . . . . . . . . . . 585

16. Meditation und Predigt über Lukas 9,51-56 für das Examen . . 599

Nachwort des Herausgebers

Anhang ..................... . . . 635

I. Zur Chronologie der Texte _. . . . . . . . . 636

II. Zeittafel 1906-1927 . . . . . 638

III. Von Bonhoeffer belegte Vorlesungen und Seminare 640

IV. Liste des unveröffentlichten Nachlasses aus dieser Zeit ...................... 643

V. Synopse der sowohl in GS als auch in DBW 9 abgedruckten Texte . . . . . . . . . . . . . . . 647

Abkürzungsverzeichnis

Literaturverzeichnis

a) Von Bonhoeffer benutzte Literatur . . . . . . . . . . . 649

b) Von Briefpartnern Bonhoeffers erwähnte Literatur 655

c) Vom Herausgeber benutzte Literatur . . . . . . . . . . . . 656 
Biogramme der Familie Bonhoeffer/von Hase . . . . 660

$\begin{array}{ll}\text { Register .. } & 667\end{array}$
a) Bibelstellen
669
b) Personen .
679
c) Sachen .
688 
